\title{
Analysis of differential gene expression caused by cervical intraepithelial neoplasia based on GEO database
}

\author{
SHENGHUI YAO $^{1}$ and TAIFENG LIU ${ }^{2}$ \\ Departments of ${ }^{1}$ Gynecology and ${ }^{2}$ Medical Oncology, The First People's Hospital of \\ Xuzhou, Xuzhou, Jiangsu 221000, P.R. China
}

Received September 5, 2017; Accepted March 6, 2018

DOI: $10.3892 / \mathrm{ol} .2018 .8403$

\begin{abstract}
The aim of the present study was to identify the differentially expressed genes between cervical intraepithelial neoplasias (CIN) and adjacent normal tissue, and to construct a protein-protein interaction (PPI) network. A CIN dataset was obtained from Gene Expression Omnibus, and data of gene expression in CIN and adjacent normal tissue were extracted from GSE64217. The differentially expressed genes were selected using software package and heat map was drawn using the 'pheatmap' package. The selected differentially expressed genes were subjected to PPI, Gene Ontology (GO) and Kyoto Encyclopedia of Genes and Genomes (KEGG) analysis using Cytoscape, Database for Annotation, Visualization and Integrated Discovery, STRING and KOBAS. In the present study, 287 genes were differentially expressed between CIN and adjacent normal tissue, of which 170 were significantly upregulated and 118 genes were significantly downregulated $(\mathrm{P}<0.00001$, fold-change $>6)$. A differential gene expression network map was constructed to show the interactions of 30 protein products encoded by differentially expressed genes using STRING software. In particular, the key gene, EGR 1, was identified using Cytoscape software. The KEGG pathway analysis revealed that the differential genes were mainly involved in several pathways, including 'glutathione metabolism', 'arachidonic acid metabolism', and 'pentose phosphate pathway'. Results of the GO analysis showed that differential genes were enriched in different subsets. Specifically, small proline-rich protein $2 \mathrm{E}$ and 3, distal-less homeobox 5, epithelial membrane protein 1 , cornifelin, periplakin, homeobox protein Hox-A13, estrogen receptor $\alpha$, transglutaminase 1 , small proline-rich protein $2 \mathrm{~A}$, $\mathrm{Rh} \mathrm{C}$ glycoprotein, tumor protein p63, TGM3, homeobox B5
\end{abstract}

Correspondence to: Dr Taifeng Liu, Department of Medical Oncology, The First People's Hospital of Xuzhou, 19 Zhongshan Bei Road, Xuzhou, Jiangsu 221000, P.R. China

E-mail: liutaifeng618@163.com

Key words: intraepithelial neoplasias, gene ontology analysis, KEGG pathway analysis, protein-protein interaction networks, differential genes and small proline-rich protein 2D were enriched in 'epithelial cell differentiation', which affected the differentiation of epithelial cells. In conclusion, 287 differentially expressed genes were identified successfully. The key gene was identified based on the results of PPI, GO and KEGG analyses, and functional annotation and pathway analysis were also performed. Our study provides the basis for further studies on the interaction among differentially expressed genes.

\section{Introduction}

Cervical cancer is a common malignant tumor (1) with an incidence of up to $1.2-4.5$ per 10,000 delivery women. The incidence of cervical cancer has shown an increasing trend in Chinese women $(2,3)$.

Cervical intraepithelial neoplasia (CIN) is a type of precancerous lesion closely related to cervical cancer. With a high incidence of up to $6.5 \%$ (4), CIN significantly impacts the development of cervical cancer. Therefore, accurate diagnosis of CIN and better understanding of the pathogenesis of this disease will definitely improve the prevention of cervical cancer (5). However, most studies mainly focused on the treatment of this disease, and studies on its pathogenesis are relatively rare $(6,7)$. In previous years, with the explosion of gene expression data, bioinformatics-based data digging for gene expression profile analysis has become a hot research field $(8,9)$.

In the present study, the bioinformatics method was applied to analyze gene expression data to identify differentially expressed genes in CIN tissue. Our study provide references for further studies on the molecular pathogenesis of CIN.

\section{Materials and methods}

Acquisition of gene expression profiling data. Gene expression profiling data with the series number GSE64217 was obtained from the the Gene Expression Omnibus (GEO) database. GSE64217 was provided by the Indian Institute of Technology Kharagpur, School of Medical Science and Technology, Multimodal Imaging and Computing for Theranostics (West Bengal, India). The data included 2 cases of CIN, of cervical squamous cell carcinoma, and 2 of normal tissues. Biopsy samples were collected during hysterectomy, and half of each sample was analyzed with optical microscopy (Olympus, 
Table I. Major differential genes.

\begin{tabular}{llcc}
\hline Gene & \multicolumn{1}{c}{$\operatorname{logFC}$} & P-value & $\begin{array}{c}\text { Adjusted } \\
\text { P-value }\end{array}$ \\
\hline SPRR $2 A$ & 17458.83345 & $6.83 \mathrm{E}-11$ & $1.35 \mathrm{E}-06$ \\
SPRR2E & 10240.24442 & $1.30 \mathrm{E}-10$ & $1.35 \mathrm{E}-06$ \\
TGM3 & 4833.325709 & $1.26 \mathrm{E}-09$ & $8.70 \mathrm{E}-06$ \\
SCGB1D2 & 5415.64156 & $2.82 \mathrm{E}-09$ & $1.26 \mathrm{E}-05$ \\
KLK13 & 2711.589154 & $3.03 \mathrm{E}-09$ & $1.26 \mathrm{E}-05$ \\
NCCRP1 & 4315.455575 & $1.11 \mathrm{E}-08$ & $3.84 \mathrm{E}-05$ \\
AKR1B10 & -5251.888875 & $1.38 \mathrm{E}-08$ & $4.09 \mathrm{E}-05$ \\
KLK12 & 1742.502479 & $1.75 \mathrm{E}-08$ & $4.54 \mathrm{E}-05$ \\
MSLN & 2480.833944 & $2.15 \mathrm{E}-08$ & $4.97 \mathrm{E}-05$ \\
RPTN & 1595.155099 & $4.20 \mathrm{E}-08$ & $8.71 \mathrm{E}-05$ \\
\hline
\end{tabular}

Tokyo, Japan) by a pathologist for histopathological confirmation and the other half was used for microarray analysis.

Pretreatment of raw data, identification of differential genes, and preparation of a heat map. Statistical analysis on chip data was performed using BRB-ArrayTools 4.3.2 Beta software. Chip data were first pre-treated using JustRMA algorithm, and filtered and normalized using median-based method. Chip data were filtered according to the following criteria: i) No less than two times of difference of median of genes should be observed in $\geq 20 \%$ of the samples when comparing the two types of samples; and ii) missed gene expression data should be $\leq 50 \%$. The filtered genes were tested with independent-samples t-test. Classification and comparison of dataset were performed with the Class comparison tool to identify differentially expressed genes between CIN and normal tissue $(\mathrm{P}<0.00001)$. Finally, a heat map was drawn using 'pheatmap' package in ' $R$ ' software, and differentially expressed genes were highlighted.

Gene Ontology (GO) enrichment analysis. Differentially expressed genes were subjected to GO enrichment analysis and functional annotation using Database for Annotation, Visualization and Integrated Discovery (DAVID) and 'Bingo' (plug-in of Cytoscape software).

DAVID analysis: DAVID (Database for Annotation, Visualization and Integration Discovery) software, which integrates all the major public bioinformatics resources, can be used to interpret genes related to biological mechanisms by providing enrichment analysis with standardized genetic terminologies. The DAVID database aims to provide rapid accessibility of heterogeneous annotation data from enriched area and enhanced biological information levels of individual genes specifically to yield a gene list by enabling high-throughput gene function analysis. DAVID database can be downloaded for free (https://david.ncifcrf.gov/).

Kyoto Encyclopedia of Genes and Genomes (KEGG) pathway analysis. KEGG pathway analysis and functional annotation for differential genes were performed using KOBAS 3.0, which is the first hypergeometric distribution-based examination software to evaluate the significance of enrichment of pathways,

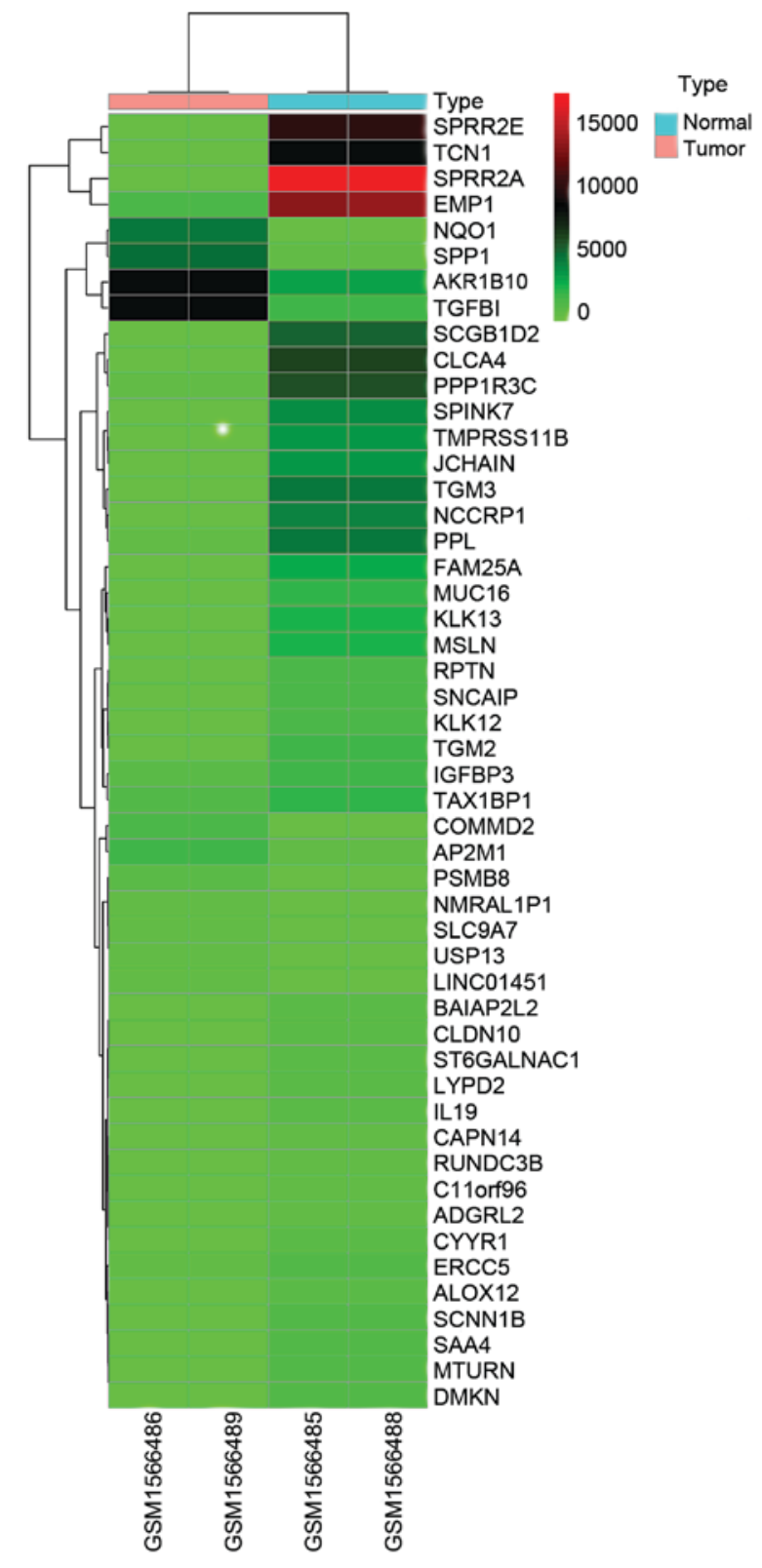

Figure 1. Heat map analysis of the first 50 differential genes. Major enrichment areas of differential genes are highlighted.

and has been successfully applied in pathway analysis for plants, animals, bacteria, and other organisms. KOBAS server can be accessed via https://kobas.cbi.pku.edu.cn.

Protein-protein interaction (PPI) network analysis. Differential genes were subjected to PPI network analysis using STRING software. PPR refers to the protein complex formed by two or more proteins through covalent bond. STRING can be accessed free of charge via https://string-db.org/.

\section{Results}

Identification of differential genes and preparation of the heat map. A total of 287 differential genes were obtained based on GSE64217, and 170 genes were significantly upregulated and 118 genes were significantly downregulated $(\mathrm{P}<0.00001$, fold-change $>6$ ). Representative differential genes are 
Table II. KEGG enrichment outcome of differential genes.

\begin{tabular}{lccr}
\hline Term & Count & P-value & FDR \\
\hline hsa00480: Glutathione metabolism & 5 & 0.006573368 & 7.715865143 \\
hsa00590: Arachidonic acid metabolism & 5 & 0.012975437 & 14.70170689 \\
hsa00030: Pentose phosphate pathway & 3 & 0.067690311 & 57.40275058 \\
hsa05230: Central carbon metabolism in cancer & 4 & 0.068191912 & 57.68095253 \\
hsa04610: Complement and coagulation cascades & 4 & 0.081430803 & 64.44748515
\end{tabular}

Term, enriched KEGG; count, number of differential genes within Term; P-value, P-value of enrichment analysis; FDR, adjusted P-value; KEGG, Kyoto Encyclopedia of Genes and Genomes; FDR, false discovery rate.

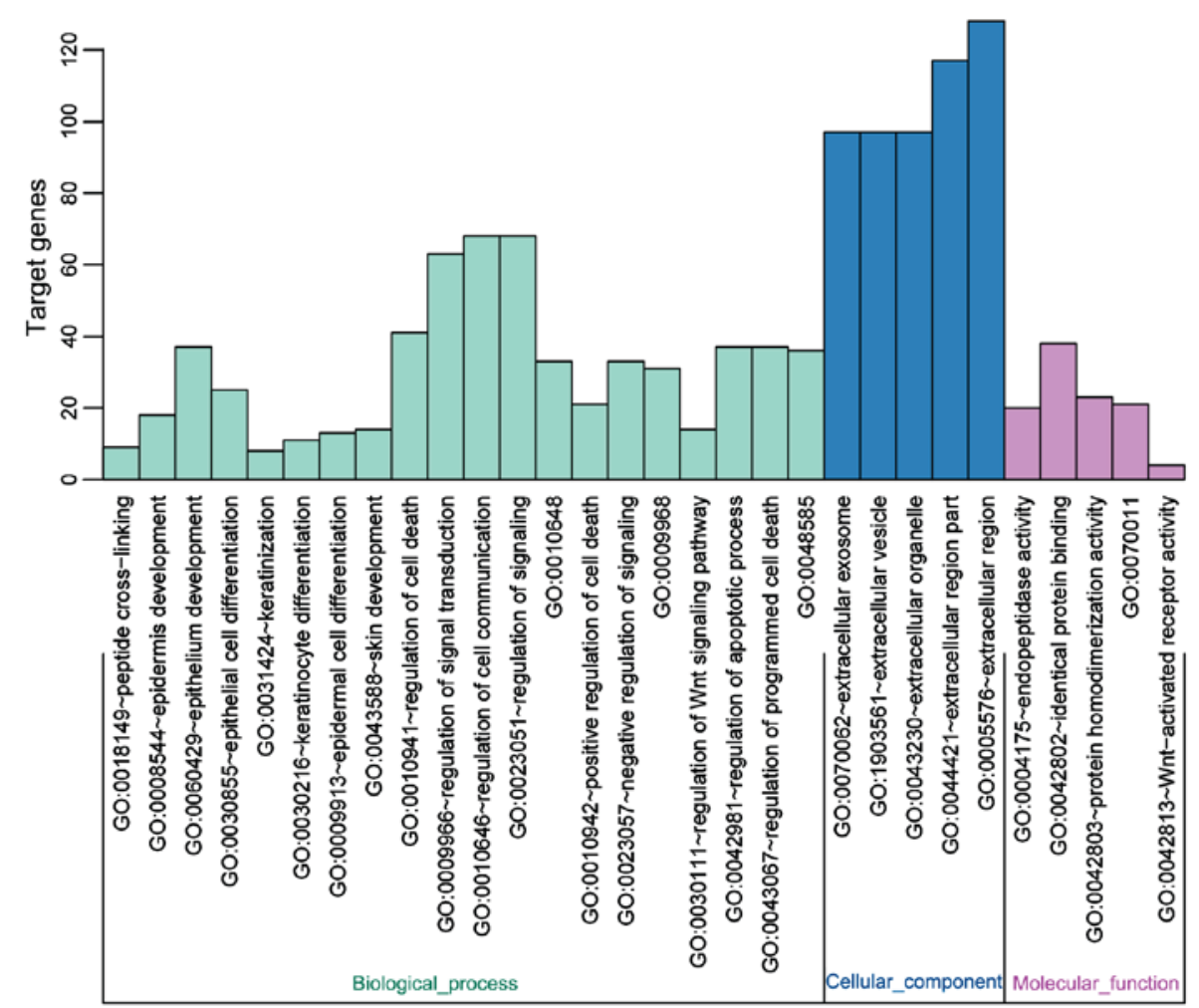

Figure 2. GO enrichment result of differential genes. The x-axis represents the enrichment of GO, and the y-axis represents the count and ratio of differential genes. Different colors correspond to different GO categories. GO, Gene Ontology.

presented in Table I. Fifty differential genes with the lowest P-values were analyzed in the heat map (Fig. 1).

GO enrichment analysis. The list of differential genes was uploaded to DAVID bioinformatics resource network (https://david.ncifcrf.gov/). The identifier was set as OFFICIAL_GENE_SYMBOL and list type as Gene List. Other parameters were all default. The results showed that differential genes were mainly concentrated in the following fields: 'Epithelial cell differentiation', 'epithelium development', and 'epidermis development', which can affect the development and differentiation of epithelial cells (Fig. 2).

KEGG pathway analysis. KOBAS 3 software was used for KEGG pathway analysis and functional annotation of differential genes, and five key KEGG pathways were identified, including 'glutathione metabolism', 'arachidonic acid metabolism', and 'pentose phosphate pathway', among which 'glutathione metabolism' and 'grachidonic acid metabolism' pathways were considered to be the two most important ones (Table II).

PPI analysis. With PPI analysis using STRING software, 30 prominent proteins were identified of which estrogen receptor $\alpha$ (ESR1), STAT1, AURKA and GAK are the relatively important. EGR1 was considered to be the most important protein and connected 14 nodes (Figs. 3 and 4).

\section{Discussion}

Cervical cancer is a common malignant tumor in women (10). In China, approximately 30,000 deaths and 100,000 new cases are reported annually (11). CIN, the precancerous lesion closely related to cervical cancer, is considered to be of great 


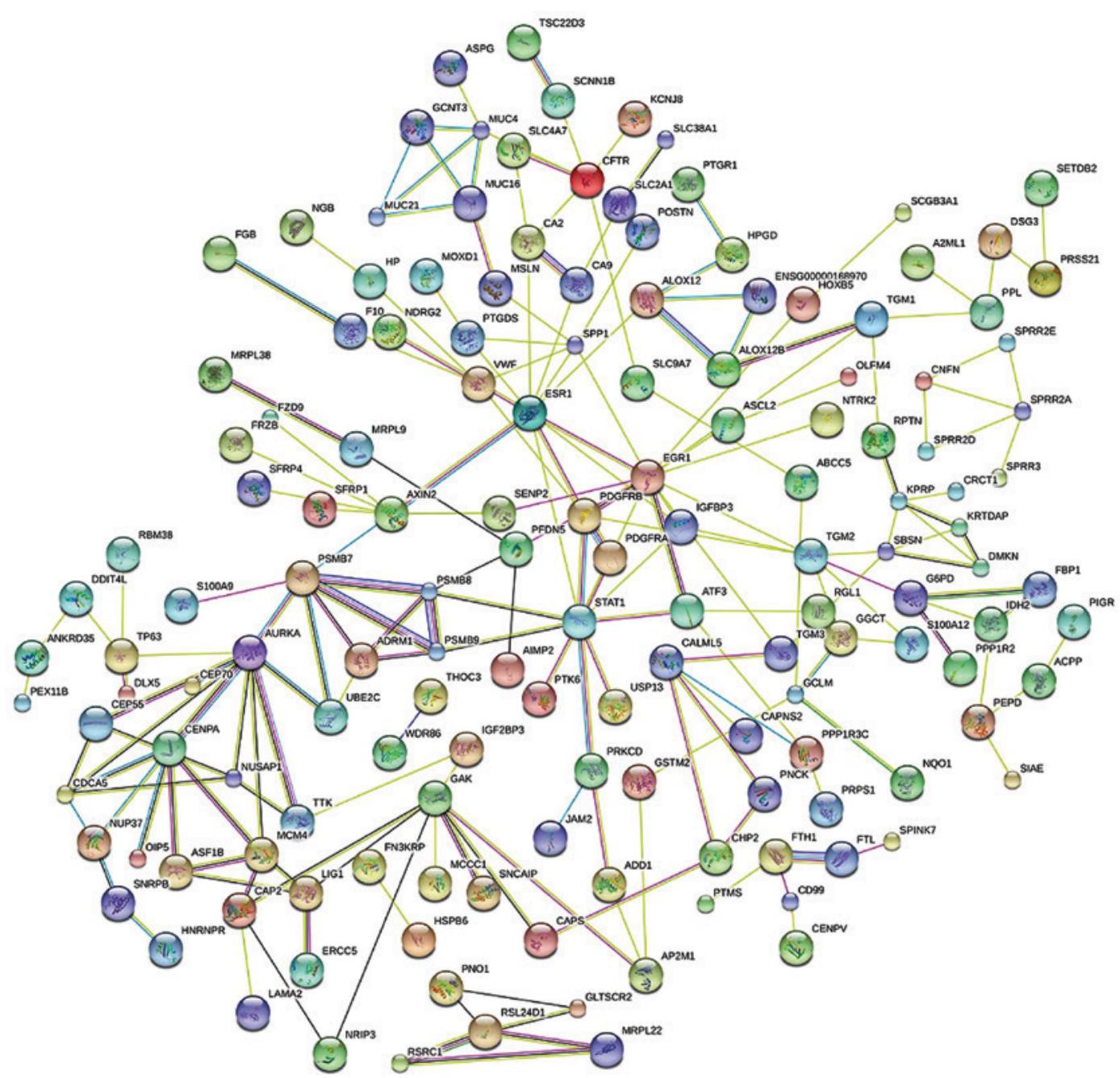

Figure 3. Protein-protein interaction (PPI) map. Circle represents the gene; line, protein-protein interaction (PPI); result in the circle, structure of the protein. Color of the arrows indicates varying PPI evidence.

significance in studies of cervical cancer $(12,13)$. Transition from CIN to cervical cancer may take as long as 10 years (14). Therefore, early diagnosis, on-time follow-up and early treatment of CIN may effectively inhibit the development of cervical cancer (15). The latest report of American Society of Clinical Oncology (ASCO) showed that the patients with CIN were becoming younger, especially for urban residents (16).

In order to investigate the molecular pathogenesis of CIN, we analyzed the differential expression between CIN patients and healthy controls using gene expression profiling data with multiple bioinformatic methods including enrichment analysis and PPI analysis. In the present study, strict inclusion criteria were followed to select the most reliable chips from microarray candidates. The reliability of the results was secured by the use of microarray data from multiple samples, which can reduce the error rate.

Based on GEO public database, we analyzed and integrated the chip data using software package, and the resulting 287 differential genes were further treated for PPI analysis with STRING. PPI analysis with differential genes showed that EGRI and ESRI genes are important factors affecting CIN. EGR1 protein is regarded as the protein with the most significant impact on CIN. Van den Brandt et al (17)

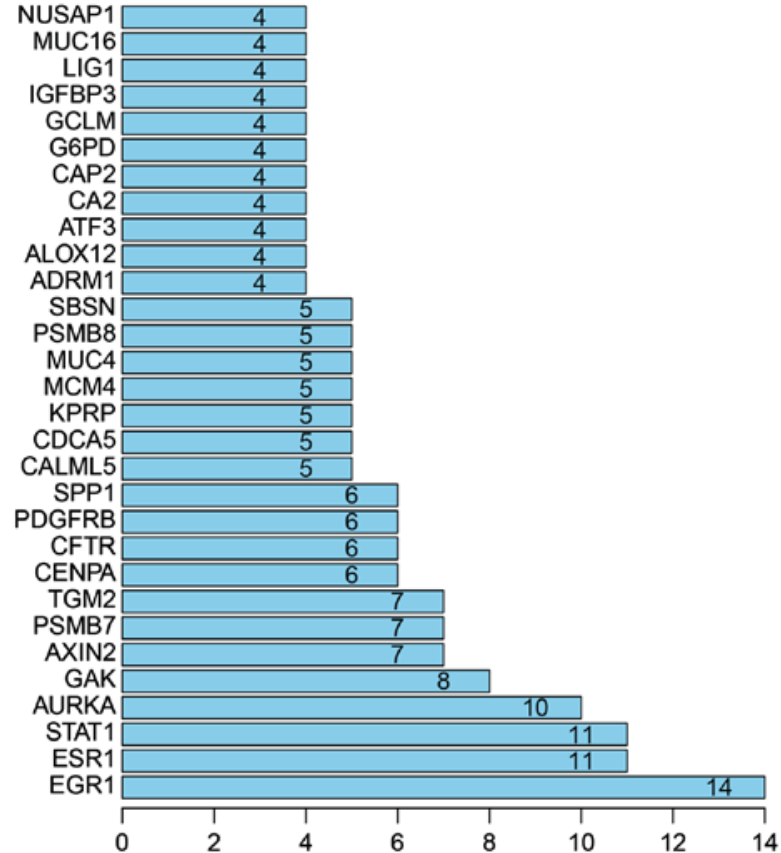

Figure 4. Histogram of key proteins. The y-axis represents the name of genes, the $\mathrm{x}$-axis represent the number of adjacent genes, and height is the number of gene connections. 
suggested that EGR1 was closely associated with the development of myopia and non-small cell lung cancer in human. EGR1 is also an important factor affecting the cell dysplasia, dedifferentiation, and synthesis of nucleoli and ribosomes (18). The activation of EGR 1 is related to the normal growth and differentiation of cells. However, cell dysplasia and dedifferentiation play an important role in the development of CIN. Therefore, EGR1 can serve as a marker for the diagnosis of CIN. Schiavon et al (19) suggested that ESR1 is a potential risk factor for breast cancer and can be used as a tumor marker for targeted therapy of breast cancer. The biological effects of ESR 1 mainly affect estrogen-targeted organs. ESR1 is mainly expressed in cytoplasm of cervical cancer cells, but not in the nucleus, possibly due to the blocked protein translation and modification. However, with the progression of CIN, the expression of ESR1 in epithelial cells is gradually declining, indicating that ESR1 can be used as marker for the early diagnosis of CIN. But further studies are needed to confirm these conclusions. GO enrichment analysis found that differential expression between CIN cancer cells and normal cells was mainly observed in epithelial cell differentiation, epithelial cell development, and epidermal development. Nagaoka et al (20) concluded that the biological processes of 'epithelial cell differentiation' and 'development' played an equally dominant role in the pathogenesis of breast and lung cancer, making the two the focus of study on the biological process of lung cancer. The specific maintenance of differentiation ability in squamous epithelial cells is an important feature of CIN, indicating the important impact of EGR1 and ESR1 on CIN. KEGG pathway analysis revealed the dominant role of glutathione metabolism and arachidonic acid metabolism pathways in CIN. A previous study carried out by Liu et al (21) suggested that glutathione metabolism was involved in varying aspects of the development of cancers by affecting the rate of cancer progression. Glutathione, which can be found in every cell in the body, plays importance roles in the maintenance of normal immune system function. Glutathione has been used widely as basic ingredient in functional foods due to its function in improving resistance and inhibiting tumorigenesis. Arachidonic acid in the human body can be synthesized by linoleic acid. The metabolism of arachidonic acid can affect cell proliferation rate, which is related to cell dysplasia of CIN. These four signal pathways also play pivotal roles in the progression of other tumors, but the correlation with CIN still has not been reported. The specific mechanism remains to be further explored.

Chip data used in this study are relatively old and sample size was relatively small. Considering that CIN-relevant genes may change with contributing factors or demographic reason (region, and ethnicity), occult genetic difference may exist. We have reduced the avoidable human error to the lowest possible level. Tumor development is difficult to predict. Further studies should focus on the gene and pathway candidates to elucidate the mechanism.

The long-term analysis led to identification that, CIN was closely related to EGRI and ESRI genes, epithelial cell differentiation and glutathione metabolism. However, the internal connection of the three factors remains to be explored with further studies. Considering the fact that only few studies on CIN-relevant genes have been reported, better understanding of CIN at the genetic level may significantly benefit the diagnosis, treatment and prognosis of CIN.

\section{Acknowledgements}

Not applicable.

\section{Funding}

No funding was received.

\section{Availability of data and materials}

The datasets used and/or analyzed during the current study are available from the corresponding author on reasonable request.

\section{Authors' contributions}

SY conceived the design of this study and wrote the manuscript. TL helped with pretreatment of raw data, identification of differential genes, and preparation of a heat map and KEGG pathway analysis. Both authors read and approved the final manuscript.

\section{Ethics approval and consent to participate}

Not applicable.

\section{Consent for publication}

Not applicable.

\section{Competing interests}

The authors declare that they have no competing interests.

\section{References}

1. Luo Q, Lei B, Liu S, Chen Y, Sheng W, Lin P, Li W, Zhu H and Shen H: Expression of PBK/TOPK in cervical cancer and cervical intraepithelial neoplasia. Int J Clin Exp Pathol 7: 8059-8064, 2014.

2. Ni L, Zheng RS, Zhang SW, Zhou XN, Zeng HM and Chen WQ: An analysis of incidence and mortality of cervical cancer in China 2003-2007. China Cancer 21: 801-804, 2012.

3. Shi YH, Wang BW, Tuokan T, Li QZ and Zhang YJ: Association between micronucleus frequency and cervical intraepithelial neoplasia grade in Thinprep cytological test and its significance. Int J Clin Exp Pathol 8: 8426-8432, 2015.

4. Jemal A, Bray F, Center MM, Ferlay J, Ward E and Forman D: Global cancer statistics. CA Cancer J Clin 61: 69-90, 2011.

5. Miller JW, Hanson V, Johnson GD, Royalty JE and Richardson LC: From cancer screening to treatment: Service delivery and referral in the National Breast and Cervical Cancer Early Detection Program. Cancer 120: 2549-2556, 2014.

6. Bueno CT, Dornelles da Silva CM, Barcellos RB, da Silva J, Dos Santos CR, Menezes JE, Menezes HS and Rossetti ML: Association between cervical lesion grade and micronucleus frequency in the Papanicolaou test. Genet Mol Biol 37: 496-499, 2014.

7. Li Q, Liu S, Liu H, Zhang J, Guo S and Wang L: Significance and implication on changes of serum squamous cell carcinoma antigen in the diagnosis of recurrence squamous cell carcinoma of cervix. Zhonghua Fu Chan Ke Za Zhi 50: 131-136, 2015 (In Chinese).

8. Rebolj M, Helmerhorst T, Habbema D, Looman C, Boer R, van Rosmalen J and van Ballegooijen M: Risk of cervical cancer after completed post-treatment follow-up of cervical intraepithelial neoplasia: Population based cohort study. BMJ 345: e6855, 2012. 
9. Luesley D and Leeson S: Colposcopy and programme management: Guidelines for the NHS Cervical Screening Programme, 2nd edition. In: SBA Questions for the MRCOG. Jones A (ed). NHSCSP Publication No. 20. Cambridge University Press, Cambridge, p126, 2010.

10. Leinonen M, Nieminen P, Kotaniemi-Talonen L, Malila N, Tarkkanen J, Laurila P and Anttila A: Age-specific evaluation of primary human papillomavirus screening vs conventional cytology in a randomized setting. J Natl Cancer Inst 101: 1612-1623, 2009

11. McCredie MR, Sharples KJ, Paul C, Baranyai J, Medley G, Jones RW and Skegg DC: Natural history of cervical neoplasia and risk of invasive cancer in women with cervical intraepithelial neoplasia 3: A retrospective cohort study. Lancet Oncol 9: 425-434, 2008

12. Hakama M, Miller AB and Day NE: Screening for cancer of the uterine cervix. Oxford University Press, UK, 2012.

13. Cuzick J, Arbyn M, Sankaranarayanan R, Tsu V, Ronco G Mayrand MH, Dillner J and Meijer CJ: Overview of human papillomavirus-based and other novel options for cervical cancer screening in developed and developing countries. Vaccine 26 : 29-41, 2008

14. Bulkmans NW, Berkhof J, Rozendaal L, van Kemenade FJ, Boeke AJ, Bulk S, Voorhorst FJ, Verheijen RH, van Groningen K, Boon ME, et al: Human papillomavirus DNA testing for the detection of cervical intraepithelial neoplasia grade 3 and cancer: 5 -year follow-up of a randomised controlled implementation trial. Lancet 370: 1764-1772, 2007.

15. Ronco G, Sideri MG and Ciatto S: Cervical intraepithelial neoplasia and higher long term risk of cancer. BMJ 335: 1053-1054, 2007.
16. Werkgroep Oncologische Gynaecologie. Cervical intraepithelial neoplasia (CIN), nationwide guidelines, 1.1: 2011 (in Dutch).

17. Van den Brandt PA, Schouten LJ, Goldbohm RA, Dorant E and Hunen PM: Development of a record linkage protocol for use in the Dutch Cancer Registry for Epidemiological Research. Int J Epidemiol 19: 553-558, 1990.

18. Van den Akker-van Marle ME, van Ballegooijen $M$ and Habbema JD: Low risk of cervical cancer during a long period after negative screening in the Netherlands. Br J Cancer 88: 1054-1057, 2003

19. Schiavon G, Hrebien S, Garcia-Murillas I, Cutts RJ, Pearson A, Tarazona N, Fenwick K, Kozarewa I, Lopez-Knowles E, Ribas R, et al: Analysis of ESR1 mutation in circulating tumor DNA demonstrates evolution during therapy for metastatic breast cancer. Sci Transl Med 7: 313ra182, 2015.

20. Nagaoka K: Zhang H, Watanabe G and Taya K: Epithelial cell differentiation regulated by McroRNA-200a in mammary glands. PLoS One 8: e65127, 2013.

21. Liu Y, Hyde AS, Simpson MA and Barycki JJ: Emerging regulatory paradigms in glutathione metabolism. Adv Cancer Res 122: 69-101, 2014

This work is licensed under a Creative Commons Attribution-NonCommercial-NoDerivatives 4.0 International (CC BY-NC-ND 4.0) License. 